\title{
Paediatric obesity prevention: The role of primary health care physicians
}

\author{
Leigh M. Vanderloo and Gillian Mandich
}

\section{University of Western Ontario}

The growing prevalence of paediatric obesity in Canada is alarming; $11.7 \%$ of children aged $5-17$ are obese. ${ }^{1}$ Given the complexity and multifaceted etiology of childhood obesity, ${ }^{2}$ preventing and treating this particular chronic disease is challenging. The following paper aims to highlight why family physicians (FPs) are ideal candidates for aiding in the prevention and management of paediatric obesity (particularly in light of the key competencies outlined by the Royal College of Physicians and Surgeons of Canada which help direct their practice). ${ }^{3}$ Recommendations for collaborating with families and multidisciplinary teams will also be provided.

\section{The role of family physicians in managing obesity}

Research suggests childhood obesity is often underrecognized and undertreated by physicians. ${ }^{4}$ Considering FPs are often the first and most frequently visited medical professionals, ${ }^{5}$ this has prompted a need for increased obesity detection and treatment in the primary care setting. A positive correlation also exists between obesity-related management training (including teaching FPs nutrition and behaviours that promote healthy weights and patientcentered counselling) and increased competence in treating patients. ${ }^{6}$ Since many FPs believe they are unprepared to manage this chronic health condition and find diagnosis difficult, ${ }^{7}$ clinical strategies that address the barriers faced by this medical authority (e.g., lack of resources, training, multidisciplinary teams, etc.) may prove useful in supporting physicians managing childhood obesity.

Specifically, physicians may benefit from regular appraisals of the literature via systematic reviews and metaanalyses, frequent attendance at academic conferences or workshops, and an increase in medical training focused on metabolic disorders; all of which will increase competency levels of FPs. In addition, innovative training methods, such as virtual learning collaborative models, have demonstrated effectiveness as reported by pediatricians in busy practice settings with limited experience and resources. ${ }^{8}$

Particularly with respect to medical training, medical schools are well-positioned to teach practitioners obesityspecific knowledge. While most medical programs adopt a biomedical approach versus a preventive one, the curriculum is not designed to tackle complex chronic health conditions like obesity; ${ }^{9}$ insufficient time is spent on weightrelated subjects in school. Providing medical students with integrated training and more community-based knowledge will motivate them to address obesity in their practice, and will also ensure soon-to-be physicians possess a strong foundation in identifying and treating this rising health concern. Such educational shifts should be given priority, not only due to the growing rates of childhood obesity, ${ }^{1}$ but because obesity along with its related co-morbidities (many of which are chronic by nature) tend to track throughout the lifespan. ${ }^{7}$ Chronic conditions are expensive to treat, and thus taxing on an already overburdened healthcare system. Consequently, taking a more preventive and health promotion-focused approach could result in long-term economic savings.

\section{Moving beyond the physician-patient dyad}

As per the abovementioned issues, most FPs believe their efforts to treat childhood obesity are ineffective. ${ }^{7}$ Therefore, it has been suggested that physicians work collaboratively with multidisciplinary teams (i.e., nutritionists, endocrinologists, researchers, exercise physiologists) to diagnose and treat obesity among their young patients. ${ }^{4}$ Due to the intricate nature of obesity, at-risk and/or obese children should not only receive a full family history, physical examination, and laboratory workup, but they should also be given lifestyle modification-focused treatment plans that promote increased physical activity, decreased screen time, and healthy diets. Accordingly, a diverse team of $\mathbf{D}$ 
c health professionals, all with respective clinical expertise, are required to design, implement, and support an 'ideal' treatment plan. ${ }^{6}$

Moreover, it is recommended that paediatric obesity prevention and treatment involve FPs as well as patients and their respective families and communities. ${ }^{10} \mathrm{It}$ is crucial that parents become active partners in their children's quest for healthy bodyweights. They not only serve as their children's primary role models, but they are also in charge of purchasing and preparing meals, as well as engaging their children in physical activities.

Conversations regarding healthy weights should begin early to promote obesity prevention as well as early detection and intervention. To fulfill their competency regarding the effective use of preventive health measures, ${ }^{3}$ it is crucial for FPs to ask parents more in-depth questions that elicit fruitful responses concerning patients' weight statuses and potential causal factors. Although related discussions may be uncomfortable and poorly received, FPs must address this subject in a respectful manner. Physicians should be mindful of their biases and potential to offend parents with the term "obesity" and/or discussions concerning the overconsumption of fast food and sweetened beverages, physical inactivity, and perceived criticism of parenting skills. ${ }^{11} \mathrm{FPs}$ should also be prepared to partner with families to develop feasible and sustainable action plans (i.e., ones that align with families' current social and financial standings), with weight loss or maintenance being the optimal goal.

\section{Future directions}

Despite ongoing efforts, increasing adiposity among Canadian children continues to be under-addressed. Given that FPs play a key role in the health of their patients, they are integral to preventing and managing childhood obesity. In terms of the role and impact FPs have in targeting and managing obesity among their young patients, their

\section{Leigh M. Vanderloo}

Leigh Vanderloo is a PhD candidate in Health and Rehabilitations Sciences (Health Promotion) at the University of Western Ontario. Her research focuses on the physical activity behaviours of preschoolers and the impact of the early learning environment on the sedentary and activity levels of young children. training-and practice-related competencies warrant further attention. ${ }^{3}$ As a means of supporting a healthier paediatric population, regularly incorporating obesity-related discourse and health promotion principles into daily practice, partnering with patients, families, and other healthcare professionals to ensure the maintenance of healthy bodyweight, as well as improving medical education, will better position FPs to combat childhood obesity.

\section{References}

1. Roberts KC, Shields M, de Groh M, Aziz A, Gilbert JA. Overweight and obesity in children and adolescents: Results from the 2009 to 2011 Canadian Health Measures Survey. Health Rep. 2012; 23(3): 37-41.

2. Colley RC, Garriguet D, Janssen I, Craig CL, Clarke J, Tremblay MS. Physical activity of Canadian children and youth: Accelerometer results from the 2007 to 2009 Canadian Health Measures Survey. Health Rep. 2011; 22(1): 15-23.

3. Royal College of Physicians and Surgeons of Canada. Can-MEDS Framework [internet]. Ottawa: Royal College of Physicians and Surgeons of Canada; 2013 [updated 2005 Mar; cited 2014 Mar 17]. Available from: http://www. royalcollege.ca/portal/page/portal/rc/canmeds/framework

4. O'Brien $\mathrm{SH}$, Holubkov R, Reis EC. Identification, evaluation, and management of obesity in an academic primary care center. Pediatrics. 2004; 14(2): e154-9.

5. Pinsonneault L, Déry V. Family physicians and treatment of pediatric obesity: Findings drawn from the creation of clinical practice guidelines. Can Fam Physician. 2011; 58(5): e238.

6. John T, Morton M, Weissman M O'Brien E,Hamburger E, Yolandra Hancock $\mathrm{Y}$, Rachel Y. Moon RY. Feasibility of a virtual learning collaborative to implement an obesity QI project in 29 pediatric practices. Int J Qual Health Care. 2014; 26(2): 205-213. doi: 10.1093/intqhc/mzu012

7. Barlow SE and the Expert Committee. Expert committee recommendations regarding the prevention, assessment, and treatment of child and adolescent overweight and obesity: Summary report. Pediatrics. 2007;120 (suppl 4): S164-92.

8. Plourde G. Preventing and managing pediatric obesity. Recommendations for family physicians. Can Fam Physician. 2006; 52: 322-8.

9. Frood, S, Johnston LM, Matteson CL, Finegood DT. Obesity, complexity, and the role of the health system. Curr Obes Rep. 2013; 2: 320-6.

10. Willms JD. Early childhood obesity: A call for surveillance and preventive measures. CMAJ. 2004; 171: 243-4.

11. Wislo VM, McGaffey A, Scopaz KA, D’Amico FJ, Jewell IK, Bridges MW, Hogan L, Hughes K. Fitwits: Preparing residency-based physicians to discuss childhood obesity with preteens. Clin Pediatr (Phila). 2013; 52(12): 1107-17.

\section{Gillian Mandich}

Gillian Mandich is presently a PhD candidate at the University of Western Ontario in Health and Rehabilitation Science, Health Promotion, where her research investigates caregiver-focused interventions to address childhood obesity. Gillian is passionate about family health, nutrition, physical activity, and diabetes research. Gillian is a yoga instructor and co-host of the podcast "The Holistic Health Diary". 